\title{
Romeo: A Tool for Analyzing Time Petri Nets
}

\author{
Guillaume Gardey ${ }^{1}$, Didier Lime ${ }^{2}$, Morgan Magnin $^{1}$, and Olivier (H.) Roux ${ }^{1}$ \\ ${ }^{1}$ IRCCyN, CNRS UMR 6597, Nantes, France \\ \{Morgan.Magnin, Guillaume.Gardey, Olivier-h.Roux\}@irccyn.ec-nantes.fr \\ ${ }^{2}$ Aalborg University - CISS, Denmark \\ didier@cs.aau.dk
}

\begin{abstract}
In this paper, we present the features of Romeo, a Time Petri Net (TPN) analyzer. The tool RomeO allows state space computation of TPN and on-the-fly model-checking of reachability properties. It performs translations from TPNs to Timed Automata (TAs) that preserve the behavioural semantics (timed bisimilarity) of the TPNs. Besides, our tool also deals with an extension of Time Petri Nets (Scheduling-TPNs) for which the valuations of transitions may be stopped and resumed, thus allowing the modeling preemption.
\end{abstract}

Keywords: Time Petri nets, model-checking, state-space, DBM, polyhedron, scheduling, stopwatch.

\section{Introduction}

Time Petri Nets (TPNs) are a classical formalism, with Timed Automata (TAs), to design reactive systems. TPNs extend classical Petri nets with temporal intervals associated with transitions. They benefit from an easy representation of real-time systems features (synchronization, parallelism ....). State reachability and boundedness is proven to be undecidable for arbitrary TPNs. However, state reachability is decidable for bounded TPNs, which is sufficient for virtually all practical purposes.

In real-time applications, it is often necessary to memorize the progress status of an action when this one is suspended then resumed. In this class of models, some extensions of Time Petri Nets have been proposed to express the preemptive scheduling of tasks. Roux and Déplanche [1] propose an extension for Time Petri Nets (Scheduling-TPNs) that consists of mapping into the Petri net model the way the different schedulers of the system activate or suspend the tasks. For a fixed priority scheduling policy, Scheduling-TPNS introduce two new attributes associated to each place that respectively represent allocation (processor or resource) and priority (of the modeled task). Bucci et al. [2] propose a similar model: Preemptive Time Petri Net (Preemptive-TPN) by mapping the scheduling policies onto transitions. 


\section{The Tool Romeo}

The purposes of the tool Romeo ${ }^{1}$ (available for Linux, MacOSX and Windows platforms) are the analysis and simulation of reactive systems modelled by TPNS or Scheduling-TPNs. It consists of: (1) a graphical user interface (GUI) (written in TCL/Tk) to edit and design TPNS, and (2) computation modules (GPN and Mercutio, written in $\mathrm{C}++$ ).

\subsection{System Design}

In a system modelling activity, the ROMEO GUI allows to model reactive systems or preemptive reactive systems using TPNs or Scheduling-TPNs. Both benefit from an easy graphical representation and from an easy representation of common real-time features (parallelism, synchronization, resources management, watch-dogs...).

As a design helper, ROMEO implements on-line simulation (TPNs, Scheduling-TPNs) and reachability model-checking (TPNS). It allows the early detection of some modeling issues during the conception stage.

\subsection{On-line Model-Checking}

In addition to on-line simulation that makes scenarii testing possible, RomEO provides an on-line model-checker for reachability. Properties over markings can be expressed and tested. It is then possible to test the reachability of a marking such that it verifies $M\left(P_{1}\right)=1 \vee M\left(P_{3}\right) \geq 3$ where $M\left(P_{i}\right)$ is the number of tokens in the place $P_{i}$ of the net. The tool returns a trace leading to such a marking if reachable.

Such a model-checker allows to verify more complex properties (quantitative properties for instance) expressed by observers (which translate a quantitative property into a reachability test), which is the main method used to study the behavior of a TPN.

\subsection{Off-line Model-Checking}

The modeling of a property using observers requires a good knowledge in TPNS and, as far as we know, no automatic observers generation is available to help a system designer.

Romeo implements different theoretical methods to translate the model analyzed into Automata, Timed Automata (TAs) or Stopwatch Automata (SWAs). The advantages of such translations is that several efficient model-checking tools are available for these models (Mec, Aldebaran, UppaAl, Kronos, Hytech). These translations also extend the class of properties that can be model-checked with observers to temporal logic (LTL, CTL) and quantitative temporal logic (TCTL).

${ }^{1}$ Download at: http://www.irccyn.ec-nantes.fr/irccyn/d/fr/equipes/TempsReel/logs 
To our knowledge, the translations of TPNS to TAs implemented in ROMEO are currently the only existing methods allowing the verification of quantitative time properties (quantitative liveness and TCTL) on TPNs.

Structural Translation. ROMEO implements a structural transformation of a TPN into a timed-bisimilar synchronized product of TAs [3] that can be modelchecked with the tool UPPAAL. The translation is optimized to take at its advantage the management of inactive clocks in UPPAAL. It follows that the algorithms implemented in this tool are used efficiently.

State Space Computation Based Translation. A first translation consists in the computation of the state class graphs (SCG) that provide finite representations for the behavior of bounded nets preserving their LTL properties [4]. For bounded TPNs the algorithm is based on DBM (Difference Bounds Matrix) data structure whereas, for Scheduling-TPNS, the semi-algorithm is based on polyhedra (using the New Polka library).

Two different methods are implemented for TPNs to generate a TA that preserves its semantics (in the sense of timed bisimilarity): the first one is derived from TA framework [5], the other one from the classical state class graph approach [6]. In the latter method, we reduce the number of clocks needed during the translation, so that the subsequent verification on the resulting TA is more efficient. In both methods, the TAs are generated in UPPAAL or KRONOs input format.

Concerning Scheduling-TPNs, the method introduced in [7] is implemented. It allows a fast translation into a Stopwatch Automaton (SWA) using an overapproximating semi-algorithm (DBM-based). Despite the overapproximation, it has been proven that the SWA is timed-bisimilar to the original SchedulingTPN. The SWA is produced in the HYTECH input format and is computed with a low number of stopwatches. Since the number of stopwatches is critical for the complexity of the verification, the method increases the efficiency of the timed analysis of the system; moreover, in some cases, it may just make the analysis possible while it would be a dead-end to model the system directly with HYTECH.

\subsection{Comparisons}

The following tables are an overview of the ROMEO features compared to two others main tools used for the analysis of TPNS and TPNS extension dealing with preemption. They compare the capabilities of the tools in terms of the properties classes that can be handled.

TINA [8] is a tool for the analysis of TPNS mainly using state class graphs techniques. ORIs [9] is a tool that analyzes Preemptive-TPNs which are equivalent to Scheduling-TPNs.

Our major contribution is to bring to TPNs frameworks (Scheduling-TPNs) methods to efficiently model-check TCTL properties. 
Table 1. RomeO capabilities on TPNS

\begin{tabular}{|c|c|c|c|c|c|}
\hline & Reachability & LTL & CTL & $\begin{array}{c}\text { Quantitative } \\
\text { Liveness }^{a}\end{array}$ & TCTL \\
\hline TINA & Marking Graph & $\begin{array}{l}\mathrm{SCG}^{b} \\
+\mathrm{MC}^{c}\end{array}$ & $\begin{array}{c}\text { Atomic SCG } \\
+\mathrm{MC}^{b}\end{array}$ & - & - \\
\hline ROMEO & $\begin{array}{l}\text { On-the-fly checking } \\
\text { or Marking Graph }\end{array}$ & $\begin{array}{c}\mathrm{SCG}+\mathrm{MC}^{c} \\
\text { or } \mathrm{ZFG}^{e}+\mathrm{MC}^{c}\end{array}$ & \multicolumn{3}{|c|}{$\begin{array}{c}\text { Translation to Timed Automata } \\
+ \text { UPPAAL }{ }^{d} \text { or KRONOS }\end{array}$} \\
\hline
\end{tabular}

${ }^{a}$ Includes response properties like $\forall \square(\varphi \Longrightarrow \forall \diamond \Psi)$ where $\varphi$ or $\Psi$ can contain clock constraints.

${ }^{b} \mathrm{SCG}=$ Computation of the State Class Graph.

${ }^{c} \mathrm{MC}=$ requires the use of a Model-Checker on the SCG or the ZFG.

${ }^{d}$ Subset of TCTL.

${ }^{e} \mathrm{ZFG}=$ Computation of the Zone-based Forward Graph.

Table 2. Romeo capabilities on Scheduling-TPNS

\begin{tabular}{|c||c|c||c|c|}
\hline \multicolumn{1}{|c||}{} & \multicolumn{2}{c||}{ State-space computation } & \multicolumn{2}{c|}{ Timed analysis } \\
\hline & Overapproximation & Exact abstraction & RTTL & TCTL \\
\hline \hline ORIS & DBM & - & $\begin{array}{c}\text { DBM-SCG }^{a} \\
+\mathrm{MC}^{b}\end{array}$ & - \\
\hline ROMEO & DBM & $\mathrm{SCG}^{c}$ & $\begin{array}{r}\text { Efficient translation to timed bisimilar } \\
\text { Stopwatch automata }+ \text { HYTECH }\end{array}$ \\
\hline
\end{tabular}

${ }^{a}$ Computation of a DBM over-approximation of the State Class Graph.

${ }^{b}$ Oris supports exact timeliness analysis of traces (with respect to a linear-time variant of Real-Time Temporal Logic (RTTL).

${ }^{c}$ As for TPNs, the SCG preserves LTL properties.

\section{Case Study}

\subsection{Description}

In this section, we work on a partial model for the control of an oscillation compensator (hydraulic shock absorber) and a differential blocking on a tractor with a sowing trailer. The partial system consists of processors running a realtime operating system, linked together with a CAN bus.

We used the translation of a Scheduling-TPN into a SWA whose state space is computed with HYTECH.

We compared the efficiency of our method with a generic direct modelling with HYTECH on this case study. We also tested several simpler and more complex related systems obtained by removing or adding tasks and/or processors. Table 3 gives the obtained results.

Columns 2 and 3 give the number of processors and tasks of the system. Columns 4, 5 and 6 describe the direct modelling in НYTECH results: the number 
of SWA used to model the system, the number of stopwatches and the time taken by HYTECH to compute the state space. For this generic modelling, we basically used the product of one SWA per task and one SWA for each scheduler. Columns 7 to 10 give the results for our method: the number of locations/transitions, stopwatches of the SWA we generated, and the time taken for its generation. Finally, the last column gives the time used by НүтесH to compute the state space of the SWA generated by our method. Times are given in seconds and NA means that the HYTECH computation could not yield a result on the machine used.

Table 3. Experimental results

\begin{tabular}{|c|c|c|c|c|c|c|c|c|c|c|}
\hline & \multicolumn{2}{|c|}{ Description } & \multicolumn{3}{|c|}{ Direct SWA Modelling } & \multicolumn{5}{|c|}{ Our method $^{a}$} \\
\hline Ex. & Proc. & Tasks & SWA's & Sw. & $t_{\text {HYTECH }}$ & Loc. & Trans. & Sw. & $t_{\text {ROMEO }}$ & $t_{\text {HYTECH }}$ \\
\hline 1 & 2 & 4 & 8 & 7 & 77.8 & 20 & 29 & 3 & $\leq 0.1$ & 0.2 \\
\hline 2 & 3 & 6 & 11 & 9 & 590.3 & 40 & 58 & 4 & $\leq 0.1$ & 0.5 \\
\hline 3 & 3 & 7 & 12 & 10 & NA & 52 & 84 & 4 & $<0.1$ & 0.7 \\
\hline 4 & $3+\mathrm{CAN}$ & 7 & 13 & 11 & NA & 297 & 575 & 7 & 0.3 & 5.3 \\
\hline 5 & $4+\mathrm{CAN}$ & 9 & 15 & 13 & $\mathrm{NA}$ & 761 & 1677 & 8 & 0.9 & 29.8 \\
\hline 6 & $5+\mathrm{CAN}$ & 11 & 17 & 15 & NA & 1141 & 2626 & 9 & 6 & 60.1 \\
\hline 7 & $6+\mathrm{CAN}$ & 14 & . & . & NA & 4587 & 12777 & 10 & 59.7 & 438.8 \\
\hline 8 & $7+\mathrm{CAN}$ & 18 & & & NA & 8817 & 25874 & 12 & 146.7 & $\mathrm{NA}$ \\
\hline
\end{tabular}

${ }^{a}$ Scheduling-TPN $\stackrel{\text { Romeo }}{\longrightarrow}$ SWA $\stackrel{\text { Hytech }}{\longrightarrow}$ state-space

These computations have been performed on a POwERPC G4 1.25GHz with $500 \mathrm{MB}$ of RAM.

We observe that the computation on a direct modelling as a product of SWA is quickly untractable (example 3). However, with our method, we are able to deal with systems of much greater size.

\section{References}

1. Roux, O.H., Déplanche, A.M.: A t-time Petri net extension for real time-task scheduling modeling. European Journal of Automation (JESA) 36 (2002) 973-987

2. Bucci, G., Fedeli, A., Sassoli, L., Vicario, E.: Time state space analysis of real-time preemptive systems. IEEE transactions on software engineering 30 (2004) 97-111

3. Cassez, F., Roux, O.H.: Structural translation from time Petri nets to timed automata. In: Fourth International Workshop on Automated Verification of Critical Systems (AVoCS'04). ENTCS, London (UK), Elsevier (2004)

4. Berthomieu, B., Diaz, M.: Modeling and verification of time dependent systems using time Petri nets. IEEE trans. on software engineering 17 (1991) 259-273

5. Gardey, G., Roux, O., Roux, O.: State space computation and analysis of time Petri nets. Theory and Practice of Logic Programming (TPLP). Special Issue on Specification Analysis and Verification of Reactive Systems (2005) to appear. 
6. Lime, D., Roux, O.H.: State class timed automaton of a time Petri net. In: 10th International Workshop on Petri Nets and Performance Models, (PNPM'03). (2003)

7. Lime, D., Roux, O.H.: A translation based method for the timed analysis of scheduling extended time Petri nets. In: The 25th IEEE RTSS'04, Lisbon, Portugal (2004)

8. Berthomieu, B., Ribet, P.O., Vernadat, F.: The tool tina - construction of abstract state spaces for petri nets and time petri nets. International Journal of Production Research 42 (2004) Tool available at http://www.laas.fr/tina/.

9. Bucci, G., Sassoli, L., Vicario, E.: Oris: A tool for state-space analysis of realtime preemptive systems. Quantitative Evaluation of Systems, First International Conference on (QEST'04) (2004) 70-79 